TITLE:

\title{
A Simple Model of Planar Membrane: An Integral Equation Investigation
}

$\operatorname{AUTHOR}(\mathrm{S})$ :

Yagi, Tomoaki; Sato, Hirofumi

\section{CITATION:}

Yagi, Tomoaki ...[et al]. A Simple Model of Planar Membrane: An Integral Equation Investigation. Journal of Computational Chemistry 2018, 39(31): 2576-2581

\section{ISSUE DATE:}

2018-12-05

URL:

http://hdl.handle.net/2433/235679

\section{RIGHT:}

This is the accepted version of the following article: TTomoaki Yagi, Hirofumi Sato. A simple model of planar membrane: An integral equation investigation. Journal of Computational Chemistry, 39(31), 2576-2581], which has been published in final form at https://doi.org/10.1002/jcc.25638. This article may be used for non-commercial purposes in accordance with Wiley Terms and Conditions for Self-Archiving:: The full-text file will be made open to the public on 28 November 2019 in accordance with publisher's 'Terms and Conditions for Self-Archiving' . This is not the published version. Please cite only the published version.; この論文は出版社版でありません。引用の際には出版社版をご確認ご利用ください。 


\title{
A simple model of planar membrane: An integral equation investigation
}

\author{
Tomoaki Yagi and Hirofumi Sato*
}

September 4, 2018

\begin{abstract}
A simple model of a lipid membrane, a binary mixture of saturated lipids and unsaturated lipids, was studied using an integral equation theory. The planar membrane is modeled as mixture of linear and bent molecules in two dimensional space, and site-site radial distribution function, Kirkwood-Buff (KB) integral and related quantities were computed over the whole range of the molar fraction to understand their mixing behavior. We found that a close packing of linear molecules is enhanced as increase the fraction of bent molecules, and a long range correlation between the linear molecules is weakened. A high concentration of linear molecules promote the demixing of linear molecules and bent molecules, and enhance the long range correlation between molecules. This implies that the higher the concentration of linear molecules, the larger clusters tend to be formed.
\end{abstract}

Keywords: $\quad$ membrane, a binary mixture, 2D, RISM

*hirofumi@moleng.kyoto-u.ac.jp, Department of Molecular Engineering, Kyoto University, Kyoto 6158510, Japan. 


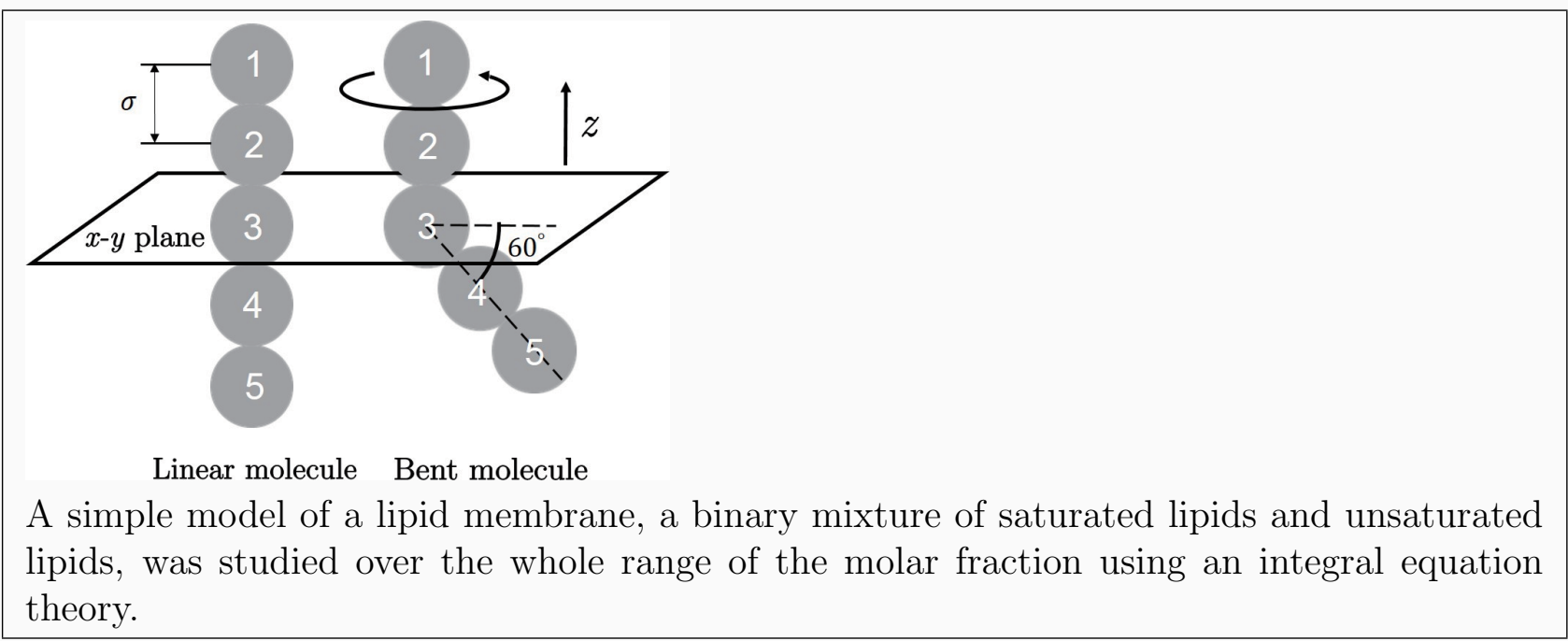




\section{INTRODUCTION}

Plasma membrane is a two dimensional liquid, which is a non-ideal mixture of large number of chemical species such as lipids, cholesterols and proteins. ${ }^{1}$ The packing of lipid molecules and their mutual affinity depend sensitively on a slight structural alteration of lipid, such as saturation of alkyl chains, leading to a complex behavior of the mixture. An example is liquid-liquid phase separation of the mixture of saturated lipids, unsaturated lipids and cholesterols. The mixture is separated into liquid-ordered (Lo) phases and liquid-disordered (Ld) phases. ${ }^{2-9}$ While the former is enriched with saturated lipids and cholesterols, the latter is with unsaturated ones. Such a phase separation occurs only below room temperature, and the mixture is almost homogeneous at physiological temperature within the resolution of the optical microscope. More microscopic, fluorescence resonance energy transfer (FRET) measurements recently reported that microdomain is formed in model membrane system even at physiological temperature, but the domain is too small to be detected by the optical microscope ${ }^{10,11}$.

Computational study is another approach to understand the microscopic structure of lipid mixture in molecular detail. Monte Carlo method for lattice model has been used to describe the phase behavior and the microdomain formation in lipid mixture ${ }^{12-16}$. Molecular dynamics (MD) simulation is also powerful tool to elucidate the atomic scale features of the membrane, and many studies have been reported targeting to understand phase separation and microdomain formation in lipid bilayer ${ }^{17-26}$. For example, Marrink and his coworkers have developed a coarse grained force field called MARTINI, specializing for lipid membrane. They applied it to a membrane consisting of saturated phosphatidylcholines (PCs), unsaturated PCs and cholesterols, showing that the spontaneous separation of the mixture into Lo and Ld phases at room temperature ${ }^{25,26}$. Recently, Straub et al. reported a comprehensive study on the system. ${ }^{27}$ Integral equation theory for fluids ${ }^{28-30}$ is an alternative approach. Thanks to the algebraic nature, fluid structure free from statistical error is provided, which could be a drawback of MD simulation. In particular, an integral equation theory for polyatomic molecular liquids called reference site interaction model (RISM) ${ }^{28-32}$ has been successfully applied to obtain structural and thermodynamic properties of various 
chemicals and biological systems ${ }^{33-35}$. The two dimensional (2D) versions of RISM equation were proposed and applied to the planar dumbbell fluid ${ }^{36-39}$. Comparison with Monte Carlo simulations showed very good agreement.

In the present study, we perform 2D RISM calculation on a simple model of lipid membrane, binary mixture of linear and bent molecules. They respectively mimic saturated and unsaturated lipids, representing the structural difference of the alkyl chain. The mixing behavior of two types molecules are elucidated in terms of local distribution of molecules, radial distribution function (RDF), and of Kirkwood-Buff (KB) integral over the whole range of the molar fraction. KB integral enables us to analyze the mutual affinity between molecules ${ }^{40-42}$, relating to thermodynamic properties of the mixture. In general RDF and KB integral are obtained by neutron and X-ray scattering experiment. ${ }^{28,43,44}$ It should be emphasized that this is the first report on binary mixture of saturated and unsaturated lipids using integral equation theory for fluids in two dimensional space. A systematic study over a wide range of molar fraction is readily achieved due to the algebraic nature of the theory.

\section{MODEL AND THEORY}

\section{Model for lipid molecule}

Lipid membrane is modeled as binary fluid mixture in a two-dimensional plane composed of linear molecules and bent molecules (Fig.1). The molecules are composed of spherical atoms to form a linear pentamer and a bent pentamer with the bond length $\sigma$. The kink of the bent molecule mimics cis double bond in the unsaturated alkyl chain that may disrupt the packing of lipid molecules. The linear molecules are always perpendicular to $x y$ plane. For bent molecules, the axis between atom 1 and 3 are perpendicular to $x y$ plane, but the 3-5 bond is tilted from $x y$ plane at $60^{\circ}$. The atom labeled with 3 is constrained on $x y$ plane and allowed to move only on this plane. The molecule can rotate only around $z$-axis, but the geometry is fixed throughout the present study. The present model is intended to grasp the essential features of mixing behavior of saturated and unsaturated lipids, over a wide range of the molar fraction. The number of interaction sites, five, is almost a minimum number to 
adequately mimic and distinguish the structural feature of these two-types of lipids.

The interaction between atoms is given by Lennard-Jones like potential

$$
u_{\alpha \beta}(r)=4 \epsilon\left[\left(\frac{\sigma}{\sqrt{r^{2}+z_{\alpha \beta}^{2}}}\right)^{12}-\left(\frac{\sigma}{\sqrt{r^{2}+z_{\alpha \beta}^{2}}}\right)^{6}\right],
$$

where $z_{\alpha \beta}$ is the difference between $z$ coordinates of sites $\alpha$ and $\beta,\left|z_{\alpha}-z_{\beta}\right|$, and $r$ is a distance between the two sites projected on $x y$ plane. Namely, the denominator represents a distance in three dimensional space, and the interaction between molecules in 3D space is effectively mapped on the two-dimensional space. The parameters $\epsilon$ and $\sigma$ are the same for all atomistic sites. The ratio of bond length to $\sigma$ is set as unity to represent the typical chemical bond.

\section{Integral equation theory}

Let us consider a binary mixture composed of $N^{\mathrm{L}}$ linear molecules and $N^{\mathrm{B}}$ bent molecule in a two-dimensional area $A$ with the total number of density $\rho=\rho^{\mathrm{L}}+\rho^{\mathrm{B}}=\left(N^{\mathrm{L}}+N^{\mathrm{B}}\right) / A=N / A$. The molar fraction of linear molecule and bent molecule are defined as $x^{\mathrm{L}}=N^{\mathrm{L}} / N$ and $x^{\mathrm{B}}=1-x^{\mathrm{L}}$, respectively.

In Fourier space, RISM integral equation in $2 \mathrm{D}$ can be written as ${ }^{36-38}$ :

$$
\mathbf{H}(k)=\boldsymbol{\Omega}(k) \mathbf{C}(k) \boldsymbol{\Omega}(k)+\boldsymbol{\Omega}(k) \boldsymbol{\rho} \mathbf{C}(k) \mathbf{H}(k),
$$

where $\mathbf{H}(k), \mathbf{C}(k)$ and $\boldsymbol{\Omega}(k)$ are matrices, whose elements are Fourier transforms of the following site-site correlation functions between interaction sites of $\alpha$ and $\beta$; intermolecular total correlation functions, $h_{\alpha \beta}(r)$, intermolecular direct correlation function, $c_{\alpha \beta}(r)$, and intramolecular correlation functions $\omega_{\alpha \beta}(r)$. $\boldsymbol{\rho}$ is diagonal matrix whose elements are densities of molecules. All of these matrices are decomposed into linear molecule part (L) and bent molecule part (B):

$$
\begin{aligned}
& \mathbf{H}(k)=\left[\begin{array}{ll}
\mathbf{H}^{\mathrm{LL}}(k) & \mathbf{H}^{\mathrm{LB}}(k) \\
\mathbf{H}^{\mathrm{BL}}(k) & \mathbf{H}^{\mathrm{BB}}(k)
\end{array}\right], \quad \mathbf{C}(k)=\left[\begin{array}{cc}
\mathbf{C}^{\mathrm{LL}}(k) & \mathbf{C}^{\mathrm{LB}}(k) \\
\mathbf{C}^{\mathrm{BL}}(k) & \mathbf{C}^{\mathrm{BB}}(k)
\end{array}\right], \\
& \boldsymbol{\Omega}(k)=\left[\begin{array}{cc}
\boldsymbol{\Omega}^{\mathrm{LL}}(k) & 0 \\
0 & \boldsymbol{\Omega}^{\mathrm{BB}}(k)
\end{array}\right], \quad \boldsymbol{\rho}=\left[\begin{array}{cc}
\rho^{\mathrm{L}} \mathbf{I} & 0 \\
0 & \rho^{\mathrm{B}} \mathbf{I}
\end{array}\right] .
\end{aligned}
$$


The structural information of molecules is described in the intramolecular correlation functions, whose Fourier-transformed expression is written as ${ }^{36-38}$,

$$
\Omega_{\alpha \beta}^{\mathrm{XX}}(k)=J_{0}\left(k l_{\alpha \beta}\right) \quad(\mathrm{X}=\mathrm{L} \text { or } \mathrm{B})
$$

where $l_{\alpha \beta}$ is a intramolecular distance between sites $\alpha$ and $\beta$ projected on $x y$ plane. Because the tilt angle is fixed, it does not depend on the rotation of bent molecule. $J_{0}$ is the zerothorder Bessel function of the first kind. Because both of functions, $c_{\alpha \gamma}$ and $h_{\alpha \gamma}$, are unknown, we need an additional relation called closure relating these functions to solve the RISM integral equation. In this study, we use PY closure approximation:

$$
g_{\alpha \beta}(r)=\exp \left[-u_{\alpha \beta}(r) /\left(k_{\mathrm{B}} T\right)\right]\left(1+h_{\alpha \beta}(r)-c_{\alpha \beta}(r)\right),
$$

where $g_{\alpha \beta}(r)=h_{\alpha \beta}+1$ is radial distribution function (RDF), and $T$ and $k_{\mathrm{B}}$ are respectively temperature and Boltzmann constant. We also employed HNC closure, but the convergence could not be obtained under some conditions.

\section{COMPUTAIONAL DETAIL}

Two-dimensional Fourier transforms in isotropic system are defined as

$$
\begin{aligned}
& \hat{f}(k)=2 \pi \int_{0}^{\infty} r f(r) J_{0}(k r) d r . \\
& f(r)=\frac{1}{2 \pi} \int_{0}^{\infty} k \hat{f}(k) J_{0}(k r) d k
\end{aligned}
$$

The right hand sides of Eqs.(5) and (6) are Fourier-Bessel transform. RISM integral Eq. (2) complemented with PY closure Eq. (4) was solved employing discrete Hankel transform on the radial grid 1000 points for $r_{\max }=30 \sigma$. The equations were converged to the root-meansquare accuracy of $10^{-6}$ by means of modified direct inversion in the iterative subspace $(\mathrm{MDIIS})^{45}$. In the calculation, the temperature and packing fraction are fixed at $T^{*}=$ $k_{\mathrm{B}} T / \epsilon=16.5$ and $\eta=\rho\left(A^{\mathrm{L}} x^{\mathrm{L}}+A^{\mathrm{B}} x^{\mathrm{B}}\right)=0.4$, where $A^{\mathrm{L}}=\pi(\sigma / 2)^{2}$ and $A^{\mathrm{B}}=3 A^{\mathrm{L}}-(\pi / 3-$ $\sqrt{3} / 4) \sigma^{2}$ are excluded areas of the linear and bent molecules, respectively. 


\section{RESULTS AND DISCUSSION}

\section{RDFs and $K B$ integrals}

Here we focus on the local distributions of linear molecules and bent molecules in the mixture.

Figure 2 plots three pairs of RDFs between the central atoms, $g_{33}^{\mathrm{LL}}(r), g_{33}^{\mathrm{LB}}(r)$, and $g_{33}^{\mathrm{BB}}(r)$, and their change with respect the molar fraction of linear molecule $\left(x^{\mathrm{L}}\right)$. Because the site dependence of RDF is negligibly small in the present computations, RDFs between other pairs are not shown. Note that $x^{\mathrm{L}}=0.0$ means an infinite dilution of linear molecule, essentially corresponding to pure bent molecule system.

As shown in the figure, the first peak of L-L RDFs at $r / \sigma \approx 1.0$ are much higher than those of L-B and B-B. It is likely that, while linear molecules preferably contact with each other, bent molecules are not in close contact because of the steric hindrance caused by the kink. The first peak of B-B RDF of pure bent molecules $\left(x^{\mathrm{L}}=0.0\right.$; solid line $)$ is lower and broader than others due to the loose packing.

Starting from pure linear molecule system $\left(x^{\mathrm{L}}=1.0\right.$; dotted line $)$ to decrease $x^{\mathrm{L}}$, the first peak of the L-L RDF becomes higher by adding bent molecules. On the other hand, the second peak $(r / \sigma \approx 2.2)$ becomes lower and lower with decrease of $x^{\mathrm{L}}$, and finally disappears at $x^{\mathrm{L}}=0$. A close packing of linear molecules is remarkably enhanced in the first neighbor by adding bent molecules. It is likely that, for the high concentration of the bent molecules, they push linear molecules into small domains. The structure of $g_{33}^{\mathrm{LL}}(r)$ in the second neighbor and beyond becomes indistinct presumably because of a lack of linear molecules as well as disruption of long range correlation. At the same time, B-B RDF is monotonically lowered in the whole range of $r$ with decreasing of $x^{\mathrm{L}}$. RDF of L-B becomes lower as $x^{\mathrm{L}}$ increases in the whole range of the distance $r$, and eventually lower than unity beyond the first-neighbor. Although inhomogeneity of fluid is not directly taken into account due to the inherent nature of the present theory, this implies that the presence of linear molecule promotes the separation of two types of molecules.

Kirkwood-Buff (KB) integral is defined as the integral of RDF over area, up to a specific 
distance $r$.

$$
G_{\alpha \beta}^{\mathrm{XY}}(r)=2 \pi \int_{0}^{r} d r^{\prime} r^{\prime}\left\{g_{\alpha \beta}^{\mathrm{XY}}\left(r^{\prime}\right)-1\right\}, \quad(\mathrm{X}, \mathrm{Y}=\mathrm{L}, \mathrm{B})
$$

quantifying the affinity between molecules in the mixture. Figure 3 presents the KB integrals between the central sites in molecules, $G_{33}^{\mathrm{LL}}(r), G_{33}^{\mathrm{LB}}(r)$ and $G_{33}^{\mathrm{BB}}(r)$. At short distances all of $\mathrm{KB}$ integrals are once decreased because of excluded volume effect. In the region where $\mathrm{RDF}$ is greater (lower) than unity, corresponding KB integral is increased (decreased).

For all $x^{\mathrm{L}}, G_{33}^{\mathrm{LL}}(r)$ is increased steeply at $r / \sigma \approx 1.0$ owing to the high, first peak, and then converged to positive value. The oscillation behaviours are seen in $2<r / \sigma<5$, especially for linear molecule-rich condition $\left(x^{\mathrm{L}}>0.6\right)$, originating from the distinct second peak (and/or beyond) in RDFs. Whilst the values of $G_{33}^{\mathrm{BB}}(r)$ and $G_{33}^{\mathrm{LB}}(r)$ are nearly zero for small $x^{\mathrm{L}}$, the former is clearly increased with $r$ for large $x^{\mathrm{L}}$. On the contrary, the decease of $G_{33}^{\mathrm{LB}}(r)$ with $r$ is enhanced because the RDF is lower than unity except the first peak. The distance at which the KB integral converges is called the correlation radius $R_{c}$, showing that there exists no correlation between molecules due to intermolecular forces beyond $R_{c}$. $R_{c}$ of $G_{33}^{\mathrm{LL}}(r)$ is increased from $R_{c} / \sigma \approx 2.0$ to $R_{c} / \sigma \approx 6.0$ as $x^{\mathrm{L}}$ is increased, indicating that long range correlation is enhanced as increasing the concentration.

\section{KB parameters}

As seen in the previous section, KB integral converges to a specific value at longer $r$. KB parameter is defined as the infinite limit of KB integrals.

$$
\overline{G^{\mathrm{XY}}}=\lim _{r \rightarrow \infty} G_{\alpha \beta}^{\mathrm{XY}}(r), \quad(\mathrm{X}, \mathrm{Y}=\mathrm{L}, \mathrm{B})
$$

Because the quantity does not depend on the site in principle, the subscript is omitted in the left hand side of the equation. The parameter gives information on the affinities between two species. For example, $\rho^{\mathrm{L}} \overline{G^{\mathrm{LL}}}$ indicates the excess value of the coordination number of linear molecules around a linear molecule with respect to the bulk region.

Figure 4 shows the molar fraction dependence of KB parameters. For small $x^{\mathrm{L}}, \overline{G^{\mathrm{BB}}}$ and $\overline{G^{\mathrm{LB}}}$ are almost similar. As linear molecules are added to the system, while $\overline{G^{\mathrm{BB}}}$ monotonically increases, $\overline{G^{\mathrm{LB}}}$ decreases up to $x^{\mathrm{L}} \approx 0.8$ and then increases. The value of $\overline{G^{\mathrm{LL}}}$ 
starts from $6.1 \sigma^{2}$ at $x^{\mathrm{L}}=0$, and increases up to $x^{\mathrm{L}} \approx 0.6$, then decreases when more linear molecules are added. This decrease is caused by the peak lowering in RDF between linear molecules as discussed previously. Basically, $\overline{G^{\mathrm{LL}}}$ and $\overline{G^{\mathrm{BB}}}$ are larger than $\overline{G^{\mathrm{LB}}}$ at any $x^{\mathrm{L}}$, meaning that the affinity between the same-type molecules is higher than that between different types. Interestingly, B-B affinity becomes larger than L-L affinity for large $x^{\mathrm{L}}$ despite the steric hindrance in bent molecules. In the large presence of linear molecules, bent molecules are segregated from the domains formed by linear molecules, and eventually their affinity looks larger.

\section{Coordination number and local molar fraction}

The coordination numbers $(\mathrm{CNs})$ of molecules $\mathrm{Y}(=\mathrm{L}, \mathrm{B})$ around molecule $\mathrm{X}(=\mathrm{L}, \mathrm{B})$ are similarly defined for site 3 as follows,

$$
N_{33}^{\mathrm{XY}}(r) \equiv \rho^{\mathrm{Y}} \int_{0}^{r} d r^{\prime} g_{33}^{\mathrm{XY}}\left(r^{\prime}\right)=\rho^{\mathrm{Y}}\left[G_{33}^{\mathrm{XY}}(r)+\pi r^{2}\right] .
$$

The boundary for the first coordination shell (CS) is chosen as the position of first local minimum $r=r_{1}$ of the RDF, and the second CS is assigned from $r=r_{1}$ to the second local minimum $r=r_{2}$. CNs for the first and second CS around linear and bent molecules are displayed in the Fig. 5. CNs for the first CS around a linear molecule are in (a), where they are monotonically increased as increasing of $x^{\mathrm{L}}$, and reach converged values, $N_{33}^{\mathrm{LL}}\left(r_{1}\right)=4.5$ and $N_{33}^{\mathrm{BL}}\left(r_{1}\right)=2.7$. The contributions from the second $\mathrm{CS}, N_{33}^{\mathrm{XY}}\left(r_{2}\right)-N_{33}^{\mathrm{XY}}\left(r_{1}\right)$, are plotted in (b), where the increase with respect to $x^{\mathrm{L}}$ is slightly rapid compared to the first CS: 7.5 for linear molecule solvent and 6.3 for bent molecule solvent. Because linear molecules are tightly packed together, CNs of linear molecules are larger than that around a bent molecule both in the first and second CSs. Fig. 5 (c) and (d) are for CNs around a bent molecule, plotted as function of $x^{\mathrm{B}}\left(=1-x^{\mathrm{L}}\right)$. As seen in the figure, $N_{33}^{\mathrm{BB}}\left(r_{1}\right)$ and $N_{33}^{\mathrm{LB}}\left(r_{1}\right)$ and those corresponding to the second CSs are nearly equal because their affinities are comparable (see Fig. 3, small $\left.x^{\mathrm{L}}\right)$. The CNs at $x^{\mathrm{B}}=1$ for the first CS are $N_{33}^{\mathrm{LB}}\left(r_{1}\right)=2.0$ and $N_{33}^{\mathrm{BB}}\left(r_{1}\right)=2.0$, and those for the second are respectively 3.4 and 3.5.

The preferential solvation around a molecule $\mathrm{X}$ is represented by the local molar fraction 
(LMF) which is defined as,

$$
\begin{aligned}
& \widetilde{x^{\mathrm{XY}}}{ }_{33}(r)=\frac{N_{33}^{\mathrm{XY}}(r)}{N_{33}^{\mathrm{XX}}(r)+N_{33}^{\mathrm{XY}}(r)} \\
& \widetilde{x^{\mathrm{XX}}}{ }_{33}(r)=\frac{N_{33}^{\mathrm{XX}}(r)}{N_{33}^{\mathrm{XX}}(r)+N_{33}^{\mathrm{XY}}(r)}=1-\widetilde{x^{\mathrm{XY}}}{ }_{33}(r) .
\end{aligned}
$$

LMF for the first CS, $\widetilde{x^{\mathrm{XY}}} 33\left(r_{1}\right)$, and that for the second $\mathrm{CS}, \widetilde{x^{\mathrm{XY}}}{ }_{33}\left(r_{2}\right)-{\widetilde{x^{\mathrm{XY}}}}_{33}\left(r_{1}\right)$, are shown in Fig. 6. The straight dashed-line in each figure indicates bulk molar fraction, namely if the plot deviates to upper side of this line, molecules tend to preferentially associate in local region, compared to the bulk. Fig. 6 (a) exhibits that both of LMF in the first and second CS are positively deviated from the bulk. The tendency is enhanced near $x^{\mathrm{L}}=0.5$ where linear and bent molecules compete in the solvation around a molecule, and $\widetilde{x^{\mathrm{LL}}}{ }_{33}\left(r_{1}\right)=0.70$ at $x^{\mathrm{L}}=0.5$ Owing to this high affinity between linear molecules, much less bent molecules come close as shown in (c).

The solvation structure around bent molecule is totally different. As shown in (b) and (d), the deviation from the bulk is relatively small. For $\widetilde{x^{\mathrm{BB}}} 33\left(r_{1}\right)$, the maximum deviation is seen in small $x^{\mathrm{B}}$ region though it is closer to the bulk, $\widetilde{x^{B B}}{ }_{33}\left(r_{1}\right)=0.58$ at $x^{\mathrm{B}}=0.5$. The linear and bent molecules prefer to be solvated by themselves rather than other species.

\section{CONCLUSIONS}

Using an integral equation theory, we analyzed the binary mixture of linear and bent molecule in planar membrane, mimicking the lipid membrane composed of saturated and unsaturated lipid. Their mixing behavior is interpreted as follows; The structural difference between linear and bent molecules prevent the complete mixing. While the linear molecules tightly packed together with each other, the kink of bent molecules interfere with association of bent molecule with other molecules. We found that a close packing of linear molecules is enhanced as increase the fraction of bent molecules, and a long range correlation between the linear molecules is weakened. 


\section{ACKNOWLEDGMENTS}

The work was financially supported in part by Grants-in-Aid for Scientific Research on Innovative Areas "Dynamical ordering of biomolecular systems for creation of integrated functions" (JP25102001, JP25102002 and JP15K21708) as well as by Grant-in-Aid for Scientific Research (B) (JP17H03009). Theoretical computations were partly performed using Research Center for Computational Science, Okazaki, Japan. All of them were supported by the Ministry of Education, Culture, Sports, Science and Technology (MEXT) Japan. 


\section{References}

1. S. J. Singer and G. L. Nicolson, Science 1972, 175, 720-731.

2. C. Dietrich, L. A. Bagatolli, Z. N. Volovyk, N. L. Thompson, M. Levi, K.Jacobson and E. Gratton, Biophys. J. 2000, 80, 1417.

3. S. L. Veatch and S. L. Keller, Phys. Rev. Lett. 2002, 89, 268101.

4. S. L. Veatch and S. L. Keller, Biophys. J. 2003, 85, 3074.

5. S. L. Veatch and S. L. Keller, Phys. Rev. Lett. 2005, 94, 148101.

6. S. L. Veatch, K. Gawrisch and S. L. Keller, Biophys. J. 2006, 90, 4428.

7. S. L. Veatch, O. Soubias, S. L. Keller, and K. Gawrisch, Proc. Natl. Acad. Sci. USA 2007, 104, 17650-17655.

8. T. Baumgart, A. T. Hammond, P. Sengupta, S. T. Hess, D. A. Holowka, B. A. Baird, and Watt W. Webb, Proc. Natl. Acad. Sci. USA 2007, 104, 3165-3170.

9. D. Lingwood, J. Ries, P. Schwille, and K. Simons, Proc. Natl. Acad. Sci. USA 2008, 105, 10005-10010.

10. J. R. Silvius, Biophy. J. 2003, 85, 1034-1045.

11. L. M. S. Loura, F. Fernandes, M. Prieto, Eur Biophys J. 2010, 39, 589-607.

12. E. Freire and B. Snyder, Biochemistry 1980, 19, 88.

13. J. Huang and G. W. Feigenson, Biophys. J. 1999, 76, 2142.

14. P. F. F. Almeida, A. Pokorny, A. Hinderliter, Biochim. Biophys. Acta 2005, $1720,1$.

15. M. L. Frazier, J. R. Wright, A. Pokorny, and P. F. F. Almeida, Biophys. J. 2007, 92, 2422.

16. P. F. F. Almeida, Biochim. Biophys. Acta 2009, $1788,72$. 
17. R. M. Venable, Y. Zhang, B. J. Hardly, R. W. Pastor, Science 1993, 262, 223-226.

18. W. Shinoda, T. Fukada, S. Okazaki, I. Okada, Chem. Phys. Lett. 1995, 232, 308-312.

19. K. Tu, M.L. Klein, D.J. Tobias, Biophys. J. 1998, 75, 2147-2156.

20. S. W. Chiu, E. Jacobsson, H.L. Scott, Biophys. J. 2001, 80, 1104-1114.

21. S. W. Chiu, S. Vasudevan, E. Jakobsson, R. J. Mashl, H. L. Scott, Biophys. J. 2003, 85, 3624-3635.

22. M. C. Pitman, F. Suits, A.D. MacKerell Jr., S. E. Feller, Biochemistry 2004, 43, 1531815328.

23. S. A. Pandit, S. Vasudevan, S. W. Chiu, R. J. Mashl, E. Jakobsson, H. L. Scott, Biophys. J. 2004, 87, 1092-1100.

24. S. A. Pandit, E. Jakobsson, H.L. Scott, Biophys. J. 2004, 87, 3312-3322.

25. S. J. Marrink, A. H. de Varies and A. E. Mark, J. Phys. Chem. B 2004, 108, 750.

26. S. J. Marrink, H. J. Risselada, S. Yefimov, D. P. Tieleman and A. H. de Vries, J. Phys. Chem. B 2007, 111, 7812-7824.

27. G. A. Pantelopulos, T. Nagai, A. Bandara, A. Panahi, and J. E. Straub, J. Chem. Phys. 2017, 147, 095101.

28. J. P. Hansen, I. R. McDonald, Theory of Simple Liquids (2nd ed.); Academic Press, London, 1986.

29. C. G. Gray and K. E. Gubbins, Theory of Molecular Fluids; Oxford University Press, New York, 1984.

30. F. Hirata, Ed. Molecular Theory of Solvation; Kluwer, Dordrecht, 2003.

31. D. Chandler, H. C. Andersen, J. Chem. Phys 1972, 57, 1930.

32. D. Chandler, J. Chem. Phys. 1973, 59, 2749. 
33. Y. Harano, T. Imai, A. Kovalenko, M. Kinoshita, and F. Hirata

34. D. Yokogawa, H. Sato, T. Imai, and S. Sakaki, J. Chem. Phys. 2009, 130, 06411.

35. N. Yoshida, J. Chem. Phys. 2014, 140, 214118.

36. J. Talbot and D. J. Tildesley, J. Chem. Phys. 1985, 83, 6419.

37. T. Urbic and C. L. Dias, J. Chem. Phys. 2014, 140, 094703.

38. T. Urbic, J. Chem. Phys. 2016, 145, 194503.

39. T. Yagi, H. Sato, Chem. Lett. 2018, 47, 901.

40. J. G. Kirkwood and F. P. Buff, J. Chem. Phys. 1951, 19, 774.

41. A. Ben-Naim, Molecular Theory of Solutions; Oxford University Press, New York, 2006.

42. A. Ben-Naim, A. M. Navarro and J. M. Leal, Phys. Chem. Chem. Phys. 2008, 10, $2451-2460$.

43. A. B. Bhatia and D. E. Thornton, Phys. Rev. 1970, B2, 3004.

44. K. Nishikawa, Chem. Phys. Lett. 1986, 132, 50.

45. A. Kovalenko, S. Ten-no, and F. Hirata, J. Comput. Chem 1999, 20, 928. 
Figure 1: The model of molecules in membrane

Figure 2: Intermolecular site-site radial distribution of linear and bent molecule system with molar fraction $x^{\mathrm{L}}=0.0,0.2,0.4,0.6,0.8$ and 1.0

Figure 3: Kirkwood-Buff integrals for the same system as Fig. 2 with molar fraction $x^{\mathrm{L}}=$ $0.0,0.2,0.4,0.6,0.8$ and 1.0 .

Figure 4: The molar fraction dependence of Kirkwood-Buff parameters.

Figure 5: The molar fraction dependence of CNs. (a) $N^{\mathrm{LL}}\left(r_{1}\right)$ and $N^{\mathrm{BL}}\left(r_{1}\right)$, (b) the second coordination for LL and BL, (c) $N^{\mathrm{LB}}\left(r_{1}\right)$ and $N^{\mathrm{BB}}\left(r_{1}\right)$, (d) the second coordination for LB and $\mathrm{BB}$

Figure 6: The local molar fraction of linear molecules around (a) a linear molecule and (b) a bent molecule, and bent molecules around (c) a linear molecule and (d) a bent molecule in the first and second CS 


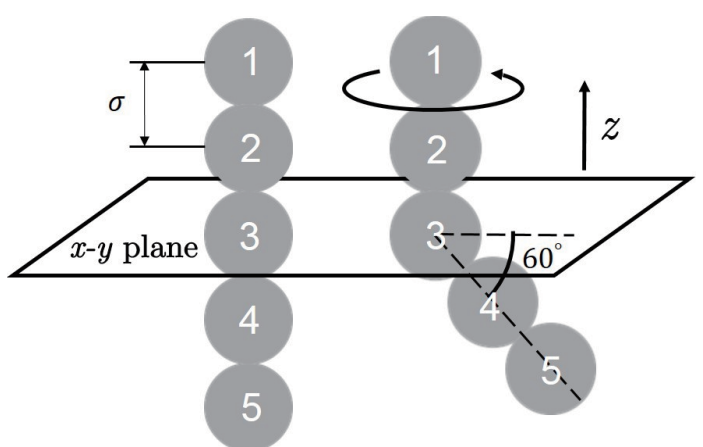

Linear molecule Bent molecule

Figure 1

Yagi and Sato J. Comput. Chem.

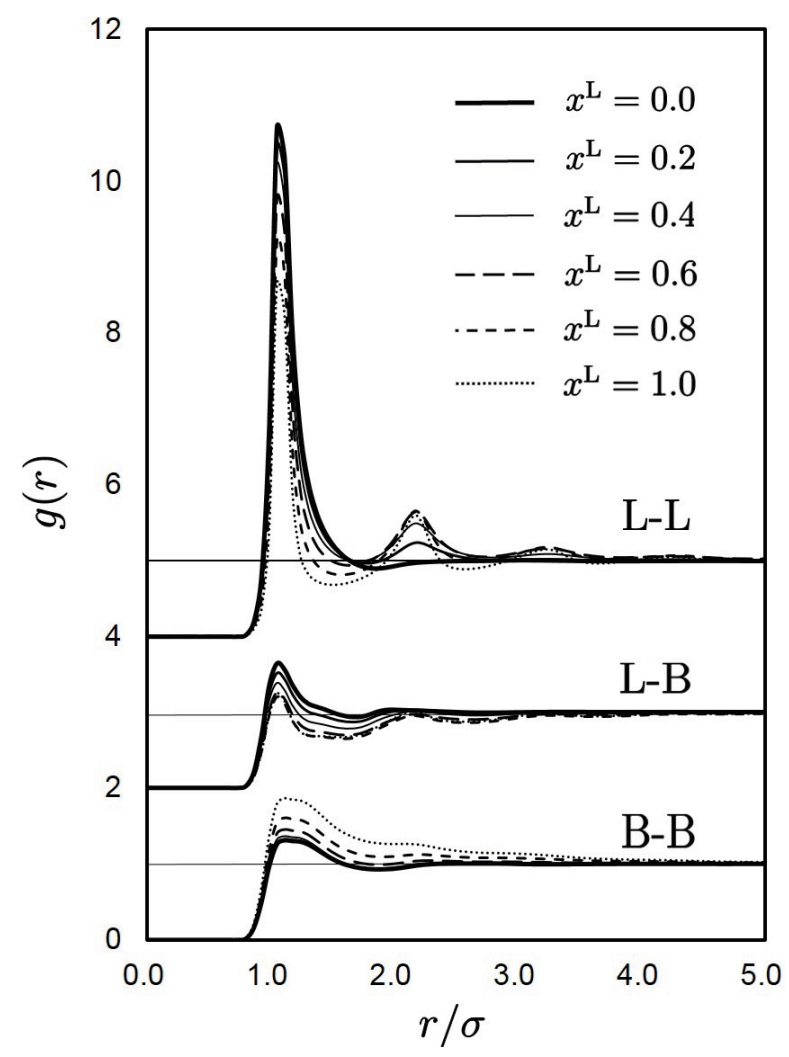

Figure 2

Yagi and Sato

J. Comput. Chem. 

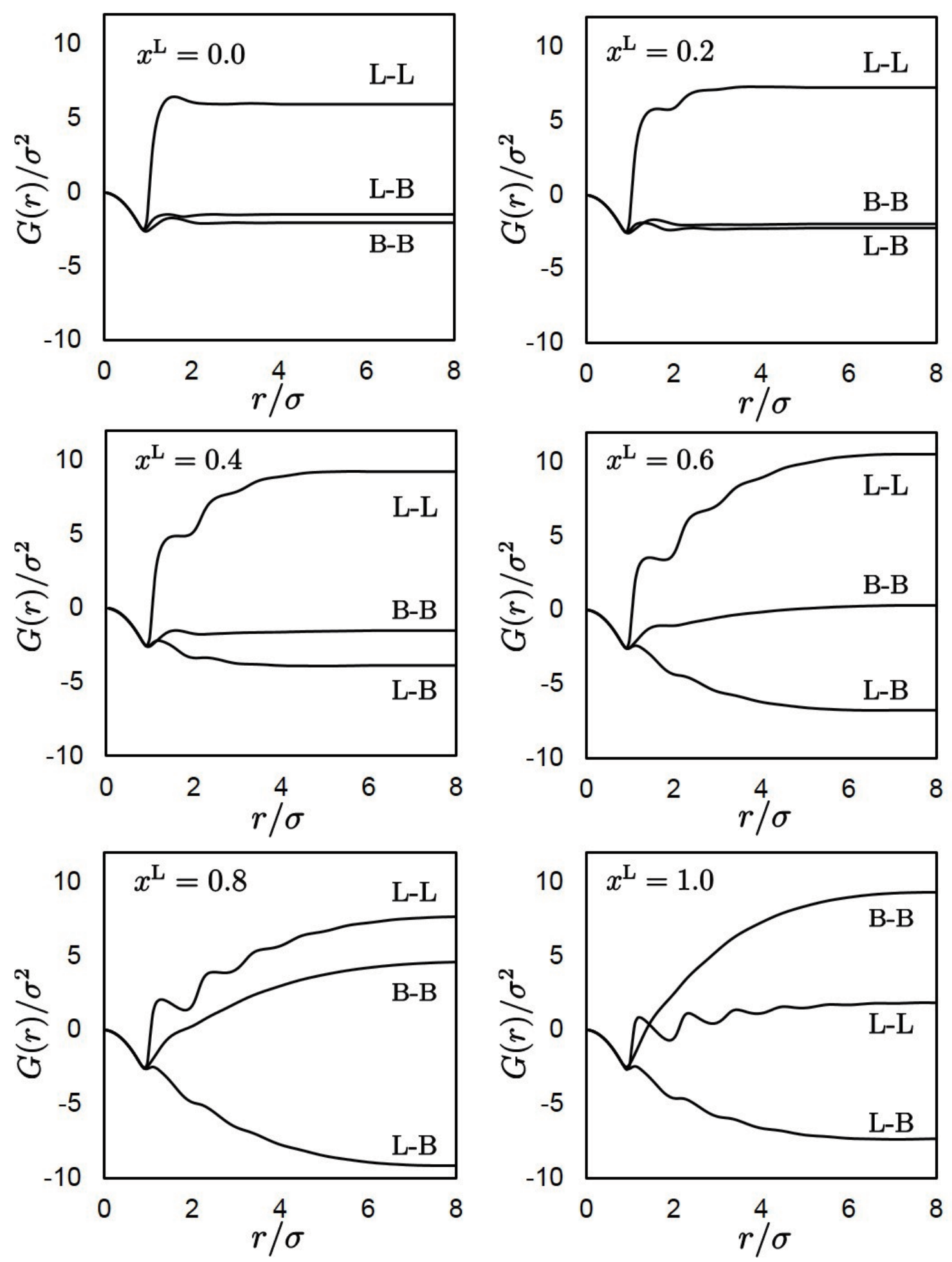

Figure 3

Yagi and Sato

J. Comput. Chem. 


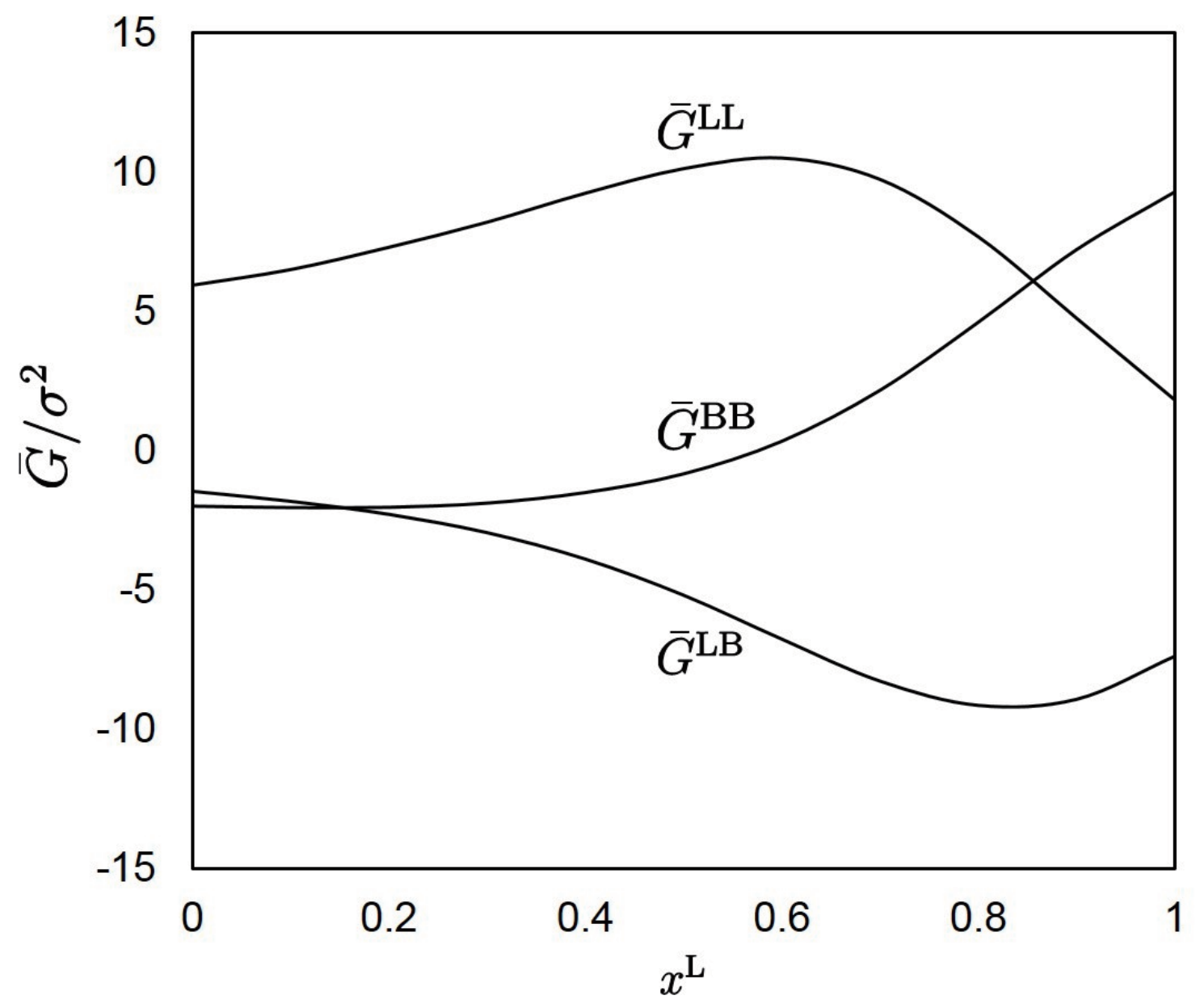

Figure 4

Yagi and Sato

J. Comput. Chem. 

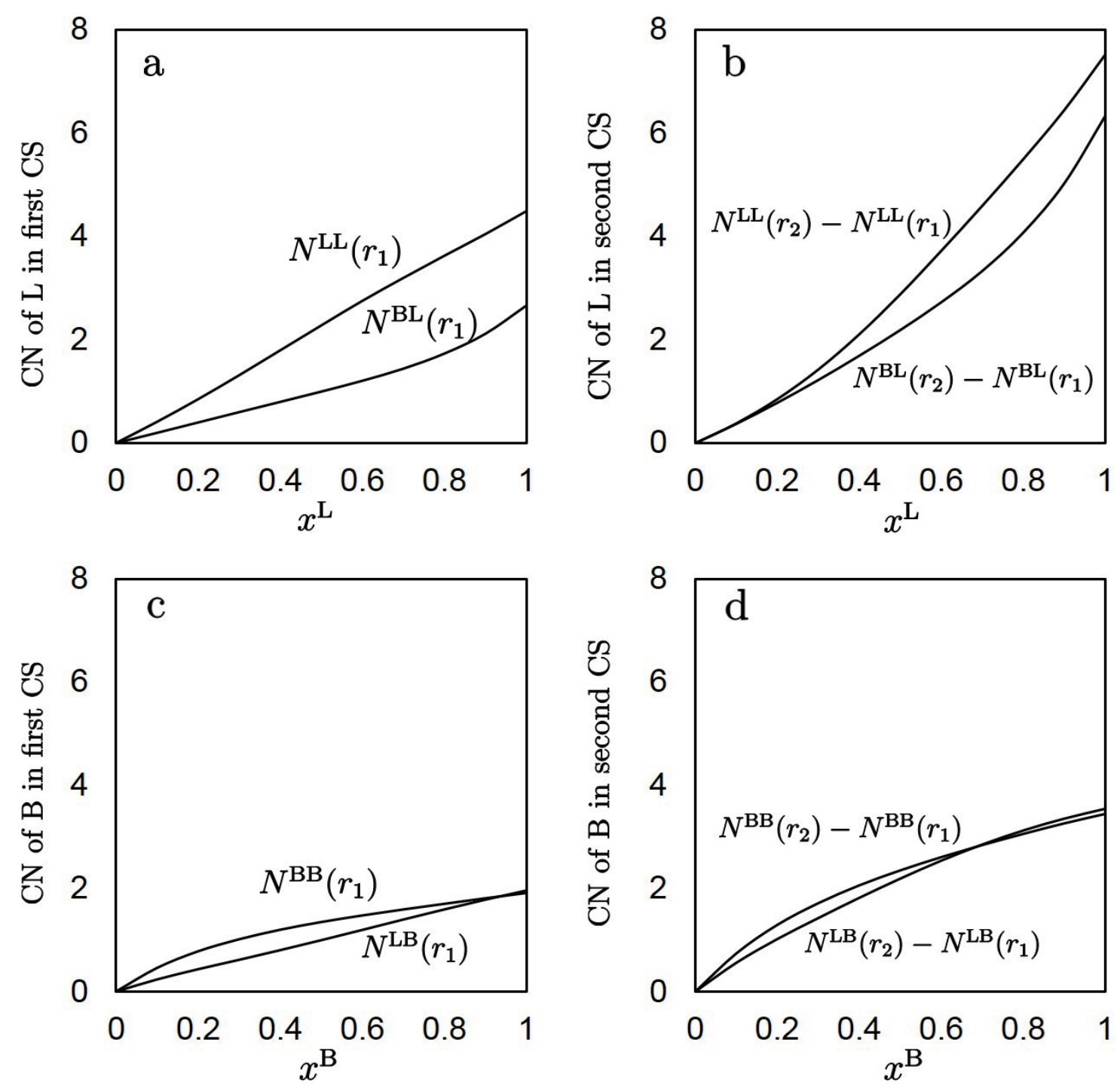

Figure 5

Yagi and Sato

J. Comput. Chem. 

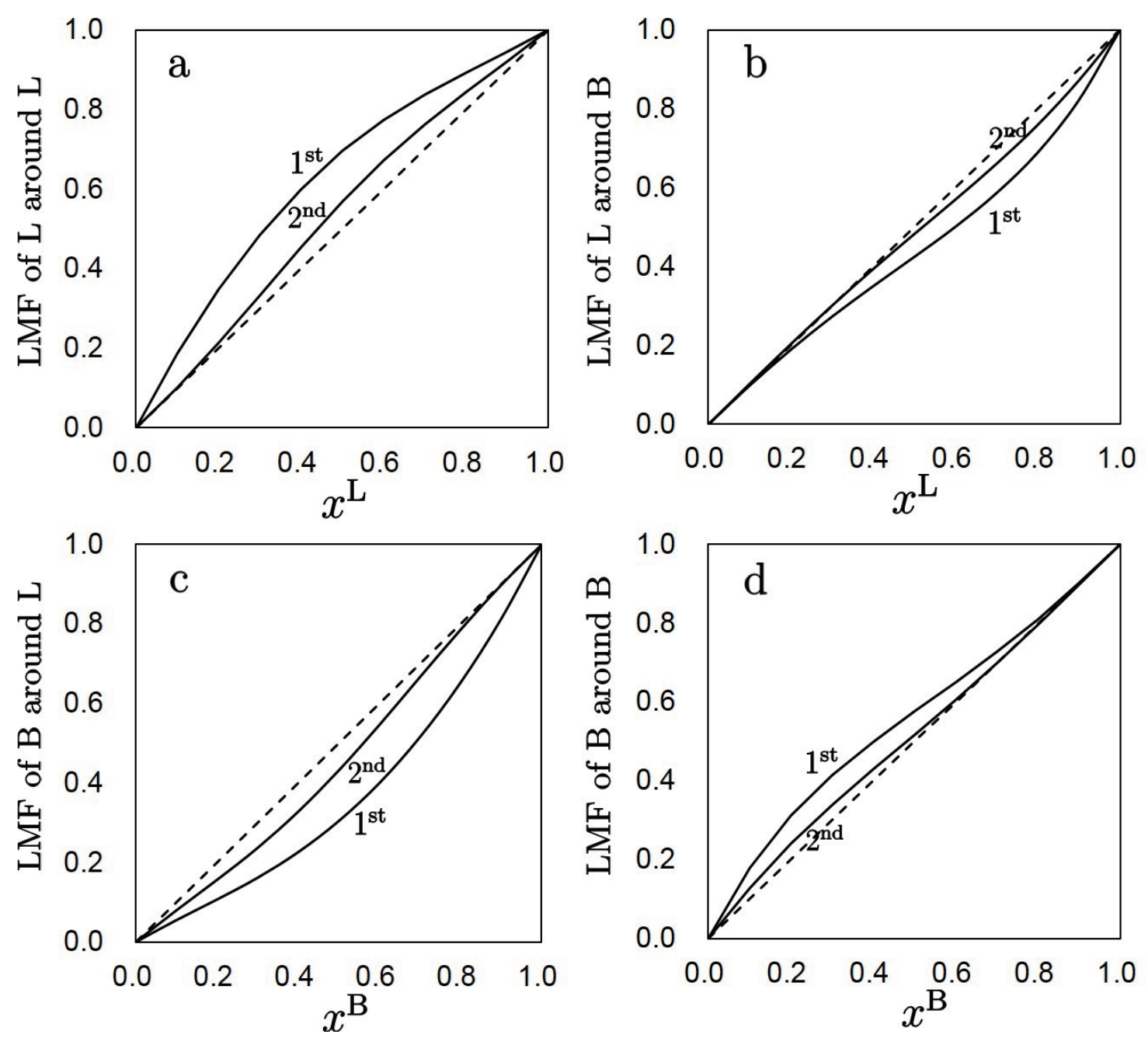

Figure 6

Yagi and Sato

J. Comput. Chem. 